\title{
Comparison of the effect of different volumes ropivacaine on deep serratus anterior plane block in patients undergoing breast surgery: a prospective randomized double-blinded trial
}

\author{
Kejian Shi ${ }^{1,2 \#}$, Ying Chen ${ }^{3 \#}$, Le Liu $^{2}$, Liangyu Zheng ${ }^{2,4}$, Lvdan Huang $^{2}$, Quanguang Wang $^{2}$, Jianping Yang $^{1,5}$ \\ ${ }^{1}$ Department of Anesthesiology, The First Affiliated Hospital of Soochow University, Suzhou, China; ${ }^{2}$ Department of Anesthesiology, The First \\ Affiliated Hospital of Wenzhou Medical University, Wenzhou, China; ${ }^{3}$ Department of Anesthesiology, Longyan First Hospital, affiliated with Fujian \\ Medical University, Longyan, China; ${ }^{4}$ Sir Run Run Shaw Hospital, Zhejiang University School of Medicine, Hangzhou, China; ${ }^{5}$ Department of \\ Anesthesiology, Dushu Lake Hospital Affiliated to Soochow University, Suzhou, China \\ Contributions: (I) Conception and design: J Yang, Q Wang; (II) Administrative support: K Shi, Y Chen; (III) Provision of study materials or patients: L \\ Liu; (IV) Collection and assembly of data: L Zheng; (V) Data analysis and interpretation: L Huang; (VI) Manuscript writing: All authors; (VII) Final \\ approval of manuscript: All authors. \\ "These authors contributed equally to this work. \\ Correspondence to: Quanguang Wang, MD. The First Affiliated Hospital of Wenzhou Medical University, South Baixiang Town, Wenzhou, China. \\ Email: wangquanguang@163.com; Jianping Yang, MD. Department of Anesthesiology, The First Affiliated Hospital of Soochow University, 188\# \\ Shizi Street, Suzhou 215006, China. Email: szyangjp@126.com.
}

\begin{abstract}
Background A larger volume of local anesthetic provides a wider range of blocked sensory but carries a greater risk. The purpose of this trial was to compare the effect of different volumes of ropivacaine injected to deep serratus anterior plane in patients undergoing breast surgery.

Methods: In this randomized double-blind trial, 60 patients undergoing breast surgery were randomly allocated to R10, R20 and R30 groups (n=20), and received deep serratus anterior plane block with 10, 20 and $30 \mathrm{~mL}$ of $0.5 \%$ ropivacaine respectively. 30 minutes after block, the cutaneous sensory was tested by cold stimulus in the craniocaudal direction along the midaxillary line. We recorded the numerical rating scale pain scores over $24 \mathrm{~h}$ after surgery and estimated the area under curve by numerical rating scale pain scores. The cases of rescue analgesia and the prevalence of adverse events were also recorded.
\end{abstract}

Results: The blocked dermatomes were $3[3,4], 6[5,7]$ and $7[6,8]$ in the R10, R20 and R30 groups, respectively ( $\mathrm{R} 10$ vs. $\mathrm{R} 20, \mathrm{P}<0.001 ; \mathrm{R} 10$ vs. $\mathrm{R} 30, \mathrm{P}<0.001 ; \mathrm{R} 20$ vs. $\mathrm{R} 30, \mathrm{P}=0.005)$. The area under curve of $\mathrm{R} 10$ group was significantly higher compared with the $\mathrm{R} 20$ and $\mathrm{R} 30$ groups $(\mathrm{P}=0.014, \mathrm{P}=0.003$, at rest; $\mathrm{P}<0.001, \mathrm{P}<0.001$, on movement).

Conclusions: The blocked dermatomes increased with increasing volume when 10, 20 and $30 \mathrm{~mL}$ ropivacaine was used for deep serratus anterior plane block. The analgesic effects of 20 and $30 \mathrm{~mL}$ were similar to each other and better than $10 \mathrm{~mL}$. Therefore, in breast surgery, volume of $20 \mathrm{~mL}$ ropivacaine is considered to be appropriate for deep serratus anterior plane block.

Keywords: Ultrasound; serratus anterior plane; anesthesia; ropivacaine

Submitted Jan 25, 2021. Accepted for publication Apr 15, 2021.

doi: 10.21037/apm-21-199

View this article at: http://dx.doi.org/10.21037/apm-21-199

^ ORCID: 0000-0003-1396-7424. 


\section{Introduction}

In recent years, interfascial plane blocks are used to reduce pain and opioid consumption for multimodal analgesia strategies (1), and serratus anterior plane (SAP) block is widely used in clinical, in which local anesthetics is injected to the fascial plane superficial or deep to the serratus anterior muscle (SAM) to block the lateral cutaneous branches of the intercostal nerves (2), thus effectively relieving postoperative pain for procedures such as breast surgery (3), thoracoscopic surgery (4), rib fractures (5) and minimally invasive heart surgery (6).

Generally, local anesthetics spreading on fascial plane depends on the volume of injection, which is related to the blocked range of sensory activity. Larger doses usually increase the risks of local anesthetics systemic toxicity. Therefore, it is always worth to explore the balance of safety and efficacy in fascial plane block.

In cadaveric studies, the dye spread in the SAP varied according to the dye volume, the site of injection, or depth of injection (superficial or deep in the SAM) (7-9). However, the fascial tissue in cadavers is different from that in the human body, and the effect of dye spread may also be different from that of local anesthetics spread. There are few reports about the relationship between the optimal volume of local anesthetics and dermatomal spread in SAP block deep to the SAM. Kunigo et al. reported that the injection of $40 \mathrm{~mL} 0.375 \%$ ropivacaine resulted in more dermatomes of sensory block than the injection of $20 \mathrm{~mL}$ $0.375 \%$ ropivacaine superficial to the SAM, but the anesthetic effects were similar across these volumes (10). However, injection deep to the SAM seems to be more recommended because it can avoid long thoracic nerve block and facilitate the operation by surgeon $(11,12)$.

Therefore, in this study, we compared the range of blocked sensory activity across different volumes of ropivacaine injection deep to the SAM. The primary endpoint was the blocked dermatomes at 30 minutes after SAP block. The secondary endpoints were the number of each blocked dermatome, the area under curve estimated by the numerical rating scale (NRS) pain scores over $24 \mathrm{~h}$ after surgery at rest and on movement, cases of rescue analgesia when NRS pain score more than 3 and the prevalence adverse events.

We present the following article in accordance with the CONSORT reporting checklist (available at http://dx.doi. org/10.21037/apm-21-199).

\section{Methods}

This is a prospective, randomized and double-blinded trial, and it was conducted in accordance with the Declaration of Helsinki (as revised in 2013). The trial was approved by the Ethics Committee at The First Affiliated Hospital of Wenzhou Medical University (No. 12, 2018) and informed consent was taken from all individual participants. The trial was registered prior to patient enrollment at the Chinese Clinical Trial Registry (ChiCTR1800015927).

\section{Enrollment}

60 patients with fibroadenoma undergoing breast surgery, aged 18 to 65 years were considered for this trial. Exclusion criteria included American Society of Anesthesiologists (ASA) physical status classification above II, body mass index (BMI) less than 18 or more than 28, peripheral neuropathy, skin pathology on the chest wall, coagulopathy, history of allergy to medications used in this study, history of substance dependence, and difficulty with communication. A random number table was used by an investigator to randomize patients into three groups: R10, R20, and R30 ( $\mathrm{n}=20$ per group), which received SAP block with 10, 20 and $30 \mathrm{~mL}$ of $0.5 \%$ ropivacaine (LBKL; AstraZeneca $\mathrm{AB}$, Sweden), respectively.

\section{Regional anesthesia}

Different volume of ropivacaine were prepared by a nurse. Patients received the SAP block prior to surgery by an anesthesiologist. Patients and anesthesiologist were unaware of treatment allocation because needle and syringe were controlled by the anesthesiologist and nurse respectively, through an extension tube connecting the needle and syringe. When patients arriving in the preoperative preparation room, standard monitors were applied and intravenous access was established. Before the SAP block, patients were asked to keeping in the lateral position. The surgical side was disinfected and covered with surgical drape. The operator used a linear probe (6 to $12 \mathrm{MHz}$; SonoSite X-Porte; SonoSite Inc., Bothell, WA, USA) to scan along the midiaxillary line and identify the fourth and fifth rib, latissimus dorsi and serratus anterior. Then the operator inserted a $22 \mathrm{G}$ needle into the plane underneath the SAM at 4-5 intercostal, using an in-plane technique, and verified the location of needle tip in the SAP through 
injecting normal saline. Then ropivacaine with different volume of $10 \mathrm{~mL}, 20 \mathrm{~mL}$ or $30 \mathrm{~mL}$ was injected into SAP from the caudal to cranial direction in R10, R20 or R30, respectively.

\section{Intraoperative management and postoperative analgesia}

When patients arrived in the operation room, standard monitors were applied. All patients in this trial received a standard regimen of general anesthesia. The anesthesiologists did not participate any procedure of this trial. After intravenous induction with propofol, sufentanil and rocuronium, and laryngeal mask airway ventilation was performed. General anesthesia was maintained with sevoflurane, propofol and remifentanil. And additional bolus of rocuronium was administrated when necessary. The depth of anesthesia monitoring was according to blood pressure, heart rare and bispectral index during surgery. Approximately 30 minutes before skin incision, tropisetron $5 \mathrm{mg}$ were administrated to prevent nausea and vomiting, and flurbiprofen $100 \mathrm{mg}$ for postoperative analgesia. Tramadol $50 \mathrm{mg}$ was administered to alleviate the postoperative pain when NRS scores was more than 3 .

\section{Data collection}

The cold test method was assessed to determine the insensitive dermatomes (13). Thirty minutes after SAP block administration, the cutaneous sensory change was tested in the craniocaudal direction along the midaxillary line. We defined a block as successful when the sensation to cold was lost or markedly reduced. NRS pain scores were recorded during rest and moving at 1, 2, 6, 12, and $24 \mathrm{~h}$ after surgery, by a researcher who unknown the group allocation. NRS pain scores on movement was assessed with a trail to lean forward from a recumbent position or a cough. We calculated the area under NRS pain scores curve by GraphPad Prism software version 7.0 (GraphPad Software Inc., San Diego, CA, USA). The presence of nausea and vomiting, rescue analgesia and other adverse events were also assessed and recorded.

\section{Statistical analysis}

All statistical analyses were carried out using SPSS statistics program version 22.0 (IBM Corporation, Armonk, NY, USA). Data were assessed for normal distribution by the Shapiro-Wilk test. Quantitative data are expressed as mean (standard deviation) or median (interquartile range), and were compared by 1-way analyses of variance or nonparametric test. Qualitative data are expressed as number (proportion), and were compared by chi-square test. Multiple comparison was tested by Mann-Whitney $\mathrm{U}$ tests. Statistical significant was considered as $\mathrm{P}<0.05$. To reduce type I error, statistical significant in post hoc multiple comparisons was correct as $\mathrm{P}<0.017$.

\section{Sample size calculation}

The sample size calculation was based on the primary endpoint of blocked dermatomes in preliminary study, and using PASS version 11.0 (NCSS Statistical Software, Kaysville, UT, USA). 15 patients were assigned to the R10, R20 and R30 groups ( $=5)$, and the blocked dermatomes were $3.2 \pm 1.10,5.4 \pm 1.14$, and $6.4 \pm 2.41$, respectively. The sample size needed in this trial was calculated as 45 patients, 15 per group, with $\alpha=0.05$ and $\beta=0.1$. To allow for possible dropouts when performing the trial, a total of 60 patients was recruited with 20 patients per group.

\section{Results}

A CONSORT statement for enrollment and allocation of patient is shown in Figure 1. There was no statistical difference between the three groups in demographic and intraoperative characteristics $(\mathrm{P}>0.05$, Table 1).

\section{Blocked dermatomes}

The blocked dermatomes were $3(3,4), 6(5,7)$ and $7(6,8)$ in the R10, R20 and R30 groups, respectively (Figure 2; $\mathrm{R} 10$ vs. $\mathrm{R} 20, \mathrm{P}<0.001 ; \mathrm{R} 10$ vs. $\mathrm{R} 30, \mathrm{P}<0.001 ; \mathrm{R} 20$ vs. $\mathrm{R} 30$, $\mathrm{P}=0.005$, respectively). The frequency of dermatomes blocked is shown in Figure 3.

\section{Pain scores}

The area under NRS pain scores curve at rest in the R10 group was significantly higher compared with the R20 and $\mathrm{R} 30$ groups [63.0 (55.0, 67.13) vs. $51.0(43.0,56.5), \mathrm{P}=0.014$; $63.0(55.0,67.13)$ vs. $44.5(32.3,57.6), \mathrm{P}=0.003$; Figure $4 A]$. The area under NRS pain scores curve on movement in the R10 group was significantly higher compared with the R20 and R30 groups [70.0 (65.0, 79.6) vs. $55.0(43.75,61.0)$, $\mathrm{P}<0.001 ; 70.0$ (65.0, 79.6) vs. 48.25 (40.0, 59.0), $\mathrm{P}<0.001$; Figure $4 B]$. However, no significant difference was found in 


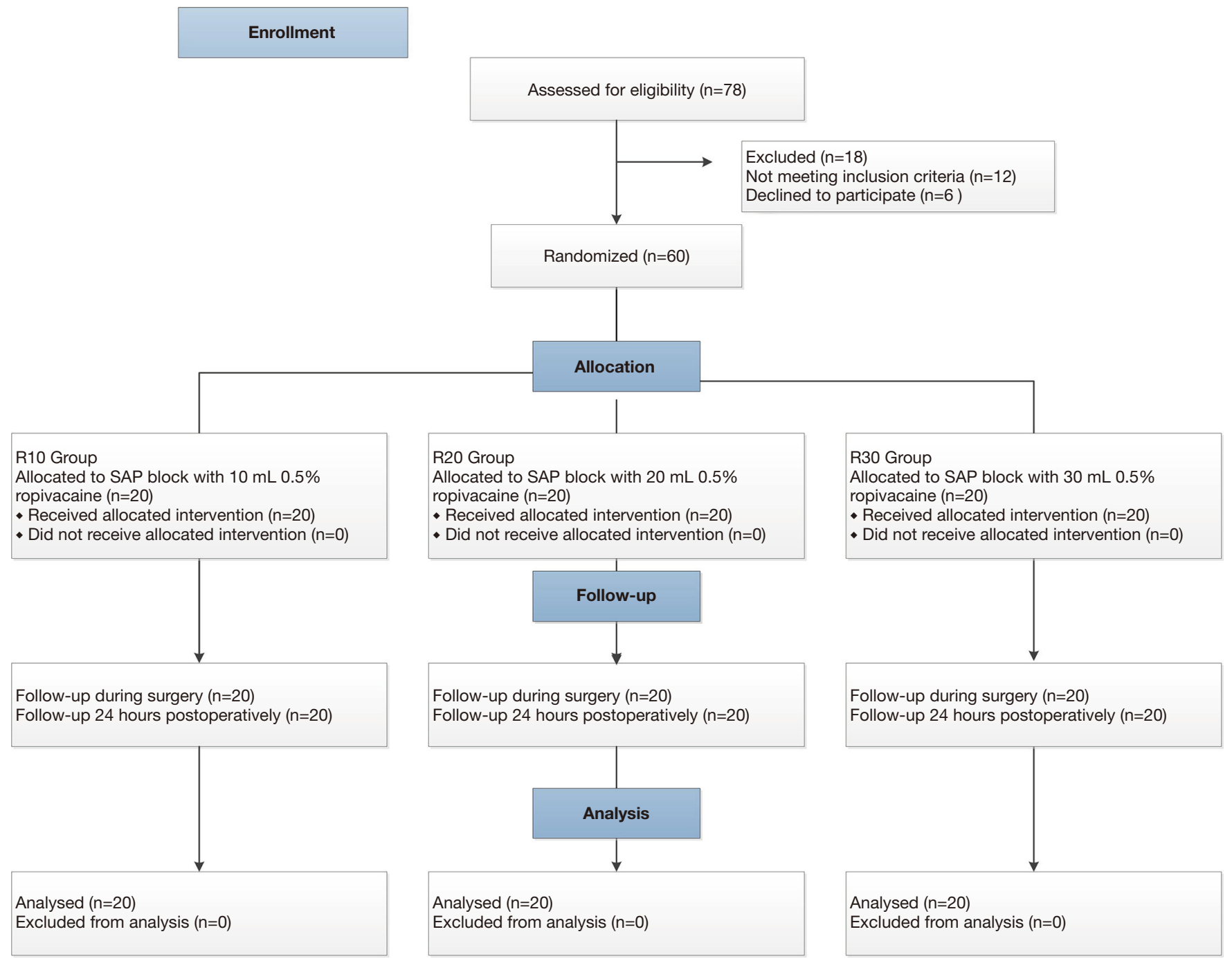

Figure 1 CONSORT flow diagram. SAP, serratus anterior plane.

Table 1 Demographic and intraoperative characteristics

\begin{tabular}{|c|c|c|c|c|}
\hline Characteristic & R10 group $(n=20)$ & R20 group $(n=20)$ & R30 group $(n=20)$ & $P$ value \\
\hline Age, y & $43.2(12.4)$ & $41.3(10.5)$ & $42.5(10.4)$ & 0.856 \\
\hline Height, cm & $159.1(2.6)$ & $157.3(4.6)$ & $160.2(4.6)$ & 0.088 \\
\hline Weight, kg & $58.4(6.8)$ & $58.5(7.3)$ & $59.9(7.5)$ & 0.781 \\
\hline Surgical duration, min & $63.8(14.0)$ & $58.3(13.0)$ & $56.8(13.4)$ & 0.785 \\
\hline Type of surgery (periareolar incision/lateral radial incision) & $2 / 18$ & $4 / 16$ & $3 / 17$ & 0.676 \\
\hline
\end{tabular}

ASA: American Society of Anesthesiologists; BMI, body mass index. Quantitative data with normal distribution are expressed as mean (standard deviation). Qualitative data are expressed as number (proportion). 


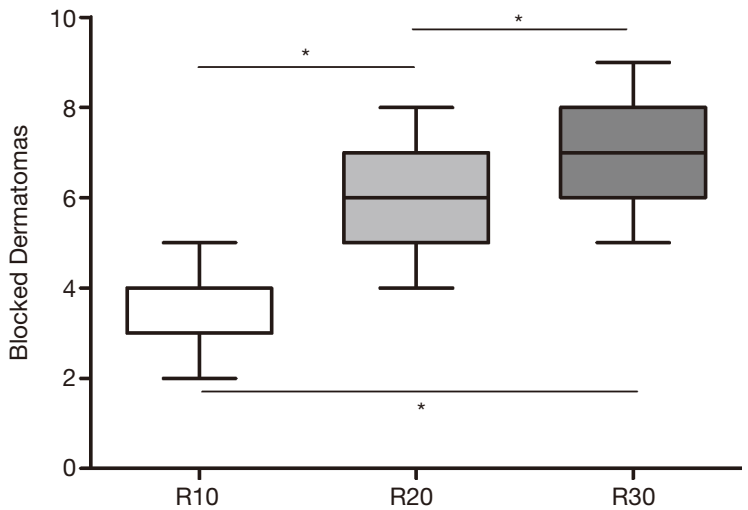

Figure 2 The blocked dermatomes at 30 minutes after SAP block. Data are presented as boxplots with median, interquartile range, minimum and maximum, $\mathrm{n}=20$. Asterisk represents a significant difference between the group comparison $(\mathrm{P}<0.017)$. SAP, serratus anterior plane.

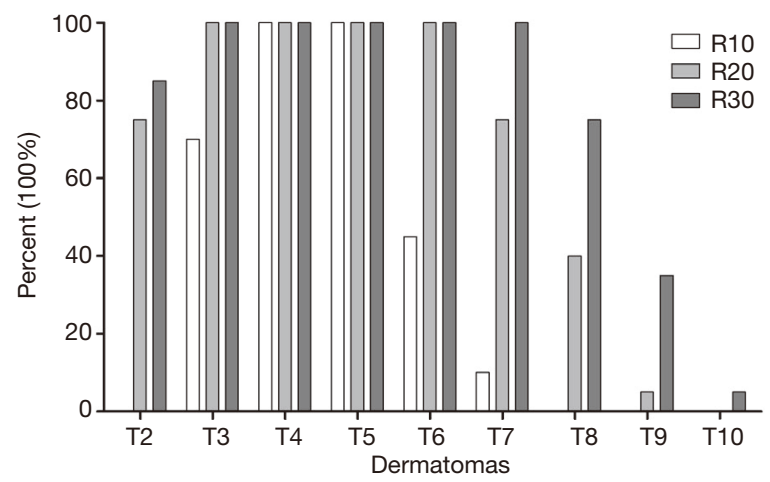

Figure 3 The frequency of dermatomes blocked at 30 minutes after SAP block. SAP, serratus anterior plane.

area under NRS pain scores curve at rest or on movement between the $\mathrm{R} 20$ and $\mathrm{R} 30$ groups $(\mathrm{P}=0.255, \mathrm{P}=0.473)$. The NRS pain scores over $24 \mathrm{~h}$ during rest and moving are shown in Figure $4 C$ and Figure $4 D$.

\section{Rescue analgesia}

Cases (rate) of rescue analgesia was $6(30 \%), 1(5 \%)$, and 2 $(10 \%)$ in the R10, R20, and R30 groups, respectively $(\mathrm{P}=0.122$, Table 2). No difference was found between the three groups.

\section{Adverse events}

No difference was found between the three groups in the prevalence (rate) of nausea and vomiting $(\mathrm{P}=0.386)$, which was 8 (40\%), $4(25 \%)$, and $6(30 \%)$ in the R10, R20, and R30 groups, respectively (Table 2). No other adverse events occurred.

\section{Discussion}

This study compared the blocked dermatomes after SAP block with volume of $10 \mathrm{~mL}, 20 \mathrm{~mL}$ and $30 \mathrm{~mL}$ of ropivacaine and found that as the injection volume increased, the blocked dermatomes also increased. Volume of $20 \mathrm{~mL} 0.5 \%$ ropivacaine achieved similar analgesia compared with volume of $30 \mathrm{~mL}$, but both were better than volume of $10 \mathrm{~mL}$.

The large amounts of local anesthetics make the analgesic effect more reliable but also increases the risk of systemic toxicity. The appropriate dose contributes to both safety and efficacy for regional anesthesia. Generally speaking, the 2 nd to the 6 th intercostal nerves innervate the sensation of the breast $(14,15)$. In this study, the results showed that volume of $20 \mathrm{~mL} 0.5 \%$ ropivacaine into the SAP at the 4-5 intercostal site blocked the lateral cutaneous branch of the 2 nd to the 6th intercostal nerves, although $30 \mathrm{~mL} 0.5 \%$ ropivacaine could block more dermatomes. Combined with the block range and the analgesic effect, $20 \mathrm{~mL} 0.5 \%$ ropivacaine met the needs of postoperative analgesia for breast surgery when compared to $30 \mathrm{~mL} 0.5 \%$ ropivacaine. Of course, it should be noted that when axillary dissection is performed, the block range should reach above the T2 level, but a single injection of 20 or $30 \mathrm{~mL}$ SAP block cannot completely reach the T2 level. The pectoral nerves-2 block may be reach the T2 level better (16).

In this study, although the blocked range of $10 \mathrm{~mL}$ ropivacaine did not provide sufficient analgesia for breast surgery, it can be suggested that three blocked dermatomes of $10 \mathrm{~mL}$ ropivacaine can be used for thoracoscopic surgery. Because the incisions in this type of surgery are usually distributed in one intercostal space, single or multiple injections of SAP block according to the incision may be more appropriate. Therefore, in a single point SAP block, the volume of local anesthetics used for postoperative analgesia may be relatively too large in thoracoscopic surgery of previous studies (17-19). The increase in blocked dermatomes does not seem to be proportional to the increase in injection volume. It may be attributed to the spreading of local anesthetics not only to the cranial-caudal direction but also to the anterior-posterior direction, which 

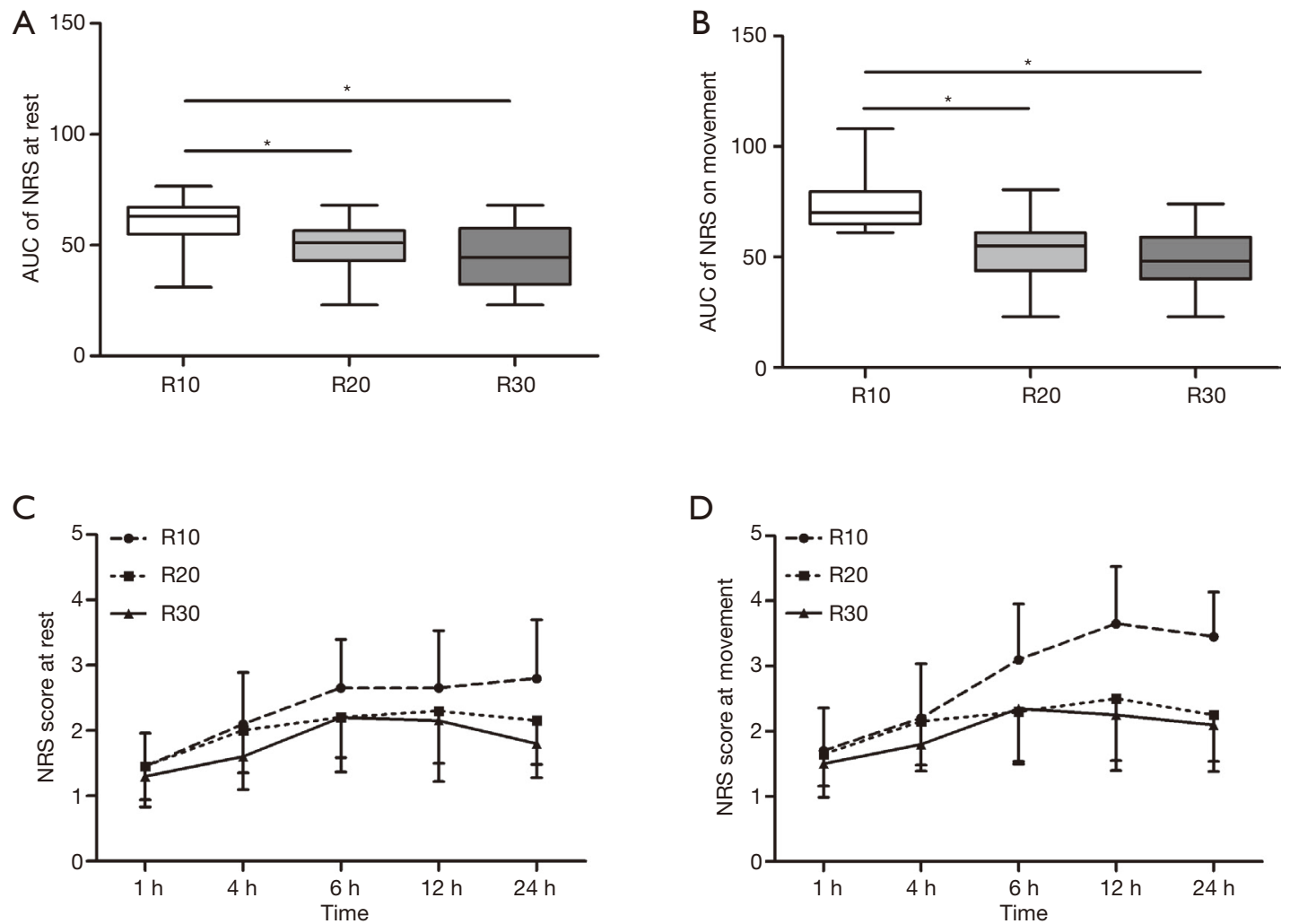

Figure 4 Record and summary of pain in 24 hours after surgery. (A) The area under NRS pain scores curve at rest over 24 h after surgery; (B) the area under NRS pain scores curve on movement over $24 \mathrm{~h}$ after surgery; (C) NRS pain scores at rest over $24 \mathrm{~h}$ after surgery; (D) NRS pain scores on movement over $24 \mathrm{~h}$ after surgery. Data in $(\mathrm{A}, \mathrm{B})$ are presented as boxplots with median, interquartile range, minimum and maximum, $n=20$. Data in (C,D) are presented as the mean (standard deviation). Asterisk represents a significant difference between the group comparison $(\mathrm{P}<0.017)$. NRS, numerical rating scale; AUC, area under curve.

has been confirmed in a cadaveric study (8). It is suggested that when a large volume of local anesthetics injection is needed to generate a large blocked range in SAP block for postoperative analgesia, multipoint injection could be considered as a method.

Although the case of rescue analgesia was found no statistical difference between the three groups, there seemed to be more cases in the R10 group than in the other two groups. It probably because the relatively small sample size could not provide the difference. Meanwhile, it also was not the primary endpoint of the study. Apart from nausea and vomiting, no other adverse events occurred, indicating that this technology was safe in this study.

The use of $0.5 \%$ and $0.75 \%$ ropivacaine was superior to $0.375 \%$ ropivacaine in postoperative analgesia for breast surgery (20). Therefore, unlike local anesthetics used in other SAP block studies, $0.5 \%$ ropivacaine was used in this study.
This study had several limitations. (I) We measured the effect of SAP block using a cold stimulus rather than a pain stimulus to assess the patient's sensitivity to cold. Børglum et al. confirmed that pain and cold testing affect similar cutaneous areas after transversus abdominis plane block, and the absence of either of these sensations can be used to confirm the presence of sensory blockade (21). (II) This trial was designed to investigate the sensory blocked range of SAP block after a single injection and did not include patients with breast cancer, who maybe suffered axillary lymph node dissection. Therefore, the blocked level must be above T2, and a single-point injection of SAP block cannot fulfill this criterion. (III) In this study, we did not measure the plasma concentration of ropivacaine. It is generally considered that neurological symptoms occurred at a mean plasma ropivacaine concentration of $2.20 \mu \mathrm{g} / \mathrm{mL}$ (22), and bilateral transversus abdominis plane block with $3 \mathrm{mg} / \mathrm{kg}$ ropivacaine have potential 
Table2 The case of rescue analgesia, nausea and vomiting

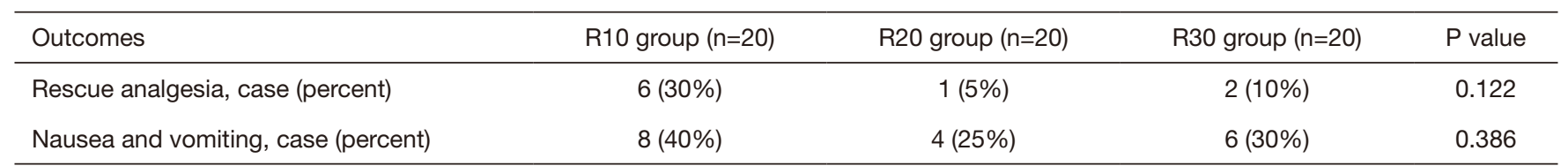

Qualitative data are expressed as number (proportion).

neurological symptoms $(23,24)$. The maximum dosage of ropivacaine in this study is $150 \mathrm{mg}$ which is closed to the dosage of $3 \mathrm{mg} / \mathrm{kg}$ on Chinese adult women weighing $50-60 \mathrm{~kg}$. However, no case of neurological symptoms was found in this study.

\section{Conclusions}

In this study, the blocked dermatomes increased with increasing local anesthetics volume when 10, 20 and $30 \mathrm{~mL} 0.5 \%$ ropivacaine was used for SAP block deep to the SAM at the 4-5 intercostal. The analgesic effects of 20 and $30 \mathrm{~mL}$ volumes were similar to each other and better than the effects of $10 \mathrm{~mL}$ volumes. Therefore, volume of $20 \mathrm{~mL}$ ropivacaine is considered to be appropriate for deep SAP block in breast surgery.

\section{Acknowledgments}

We would like to thank American Journal Experts (https:// www.aje.com/) for editing the language of this manuscript. Funding: This work was supported by grants from the National Natural Science Foundation of China [Grant No. 81900231] to Kejian Shi; Startup Fund for scientific research, Fujian Medical University, China [Grant No. 2016QH095] to Ying Chen; and Wenzhou Science and Technology Bureau, China [Grant No. Y2020778] to Kejian Shi.

\section{Footnote}

Reporting Checklist: The authors have completed the CONSORT reporting checklist. Available at http://dx.doi. org/10.21037/apm-21-199

Data Sharing Statement: Available at http://dx.doi. org/10.21037/apm-21-199

Peer Review File: Available at http://dx.doi.org/10.21037/ apm-21-199
Conflicts of Interest: All authors have completed the ICMJE uniform disclosure form (available at http:// dx.doi.org/10.21037/apm-21-199). KS reports funding from National Natural Science Foundation of China and Wenzhou Science and Technology Bureau, China. YC reports funding from Startup Fund for scientific research, Fujian Medical University, China. The other authors have no conflicts of interest to declare.

Ethical Statement: The authors are accountable for all aspects of the work in ensuring that questions related to the accuracy or integrity of any part of the work are appropriately investigated and resolved. The trial was conducted in accordance with the Declaration of Helsinki (as revised in 2013). The trial was approved by the Ethics Committee at The First Affiliated Hospital of Wenzhou Medical University (No. 12, 2018) and informed consent was taken from all individual participants.

Open Access Statement: This is an Open Access article distributed in accordance with the Creative Commons Attribution-NonCommercial-NoDerivs 4.0 International License (CC BY-NC-ND 4.0), which permits the noncommercial replication and distribution of the article with the strict proviso that no changes or edits are made and the original work is properly cited (including links to both the formal publication through the relevant DOI and the license). See: https://creativecommons.org/licenses/by-nc-nd/4.0/.

\section{References}

1. ElHawary H, Joshi GP, Janis JE. Practical Review of Abdominal and Breast Regional Analgesia for Plastic Surgeons: Evidence and Techniques. Plast Reconstr Surg Glob Open 2020;8:e3224.

2. Blanco R, Parras T, McDonnell JG, et al. Serratus plane block: a novel ultrasound-guided thoracic wall nerve block. Anaesthesia 2013;68:1107-13.

3. Mazzinari G, Rovira L, Casasempere A, et al. Interfascial block at the serratus muscle plane versus conventional 
analgesia in breast surgery: a randomized controlled trial. Reg Anesth Pain Med 2019;44:52-8.

4. Park MH, Kim JA, Ahn HJ, et al. A randomised trial of serratus anterior plane block for analgesia after thoracoscopic surgery. Anaesthesia 2018;73:1260-4.

5. Rose P, Ramlogan R, Sullivan T, et al. Serratus anterior plane blocks provide opioid-sparing analgesia in patients with isolated posterior rib fractures: a case series. Can J Anaesth 2019;66:1263-4.

6. Berthoud V, Ellouze O, Nguyen M, et al. Serratus anterior plane block for minimal invasive heart surgery. BMC Anesthesiol 2018;18:144.

7. Biswas A, Castanov V, Li Z, et al. Serratus Plane Block: A Cadaveric Study to Evaluate Optimal Injectate Spread. Reg Anesth Pain Med 2018;43:854-8.

8. Mayes J, Davison E, Panahi P, et al. An anatomical evaluation of the serratus anterior plane block. Anaesthesia 2016;71:1064-9.

9. Kunigo T, Murouchi T, Yamamoto S, et al. Spread of injectate in ultrasound-guided serratus plane block: a cadaveric study. JA Clin Rep 2018;4:10.

10. Kunigo T, Murouchi T, Yamamoto S, et al. Injection Volume and Anesthetic Effect in Serratus Plane Block. Reg Anesth Pain Med 2017;42:737-40.

11. Fuzier R, Despres C. Serratus Plane Block: New Insights But Still Many Questions. Reg Anesth Pain Med 2018;43:644.

12. Torre PA, Jones JW, Jr., Alvarez SL, et al. Axillary local anesthetic spread after the thoracic interfacial ultrasound block - a cadaveric and radiological evaluation. Rev Bras Anestesiol 2017;67:555-64.

13. Chen Y, Shi K, Xia Y, et al. Sensory Assessment and Regression Rate of Bilateral Oblique Subcostal Transversus Abdominis Plane Block in Volunteers. Reg Anesth Pain Med 2018;43:174-9.

14. Jaspars JJ, Posma AN, van Immerseel AA, et al. The cutaneous innervation of the female breast and nippleareola complex: implications for surgery. Br J Plast Surg 1997;50:249-59.

Cite this article as: Shi K, Chen Y, Liu L, Zheng L, Huang L, Wang Q, Yang J. Comparison of the effect of different volumes ropivacaine on deep serratus anterior plane block in patients undergoing breast surgery: a prospective randomized doubleblinded trial. Ann Palliat Med 2021;10(6):6104-6111. doi: 10.21037/apm-21-199
15. Sarhadi NS, Shaw-Dunn J, Soutar DS. Nerve supply of the breast with special reference to the nipple and areola: Sir Astley Cooper revisited. Clin Anat 1997;10:283-8.

16. Blanco R, Fajardo M, Parras Maldonado T. Ultrasound description of Pecs II (modified Pecs I): a novel approach to breast surgery. Rev Esp Anestesiol Reanim 2012;59:470-5.

17. Kim DH, Oh YJ, Lee JG, et al. Efficacy of UltrasoundGuided Serratus Plane Block on Postoperative Quality of Recovery and Analgesia After Video-Assisted Thoracic Surgery: A Randomized, Triple-Blind, Placebo-Controlled Study. Anesth Analg 2018;126:1353-61.

18. Shariat A, Bhatt H. Successful Use of Serratus Plane Block as Primary Anesthetic for Video-Assisted Thoracoscopic Surgery (VATS)-Assisted Pleural Effusion Drainage. J Cardiothorac Vasc Anesth 2018;32:e31-2.

19. Viti A, Bertoglio P, Zamperini M, et al. Serratus plane block for video-assisted thoracoscopic surgery major lung resection: a randomized controlled trial. Interact Cardiovasc Thorac Surg 2020;30:366-72.

20. Huang L, Zheng L, Wu B, et al. Effects of Ropivacaine Concentration on Analgesia After Ultrasound-Guided Serratus Anterior Plane Block: A Randomized DoubleBlind Trial. J Pain Res 2020;13:57-64.

21. Borglum J, Jensen K, Christensen AF, et al. Distribution patterns, dermatomal anesthesia, and ropivacaine serum concentrations after bilateral dual transversus abdominis plane block. Reg Anesth Pain Med 2012;37:294-301.

22. Knudsen K, Beckman Suurkula M, Blomberg S, et al. Central nervous and cardiovascular effects of i.v. infusions of ropivacaine, bupivacaine and placebo in volunteers. Br J Anaesth 1997;78:507-14.

23. Griffiths JD, Barron FA, Grant S, et al. Plasma ropivacaine concentrations after ultrasound-guided transversus abdominis plane block. Br J Anaesth 2010;105:853-6.

24. Toju K, Shiraishi K, Hakozaki T, et al. Plasma ropivacaine concentration following ultrasound-guided subcostal transversus abdominis plane block in adults. J Anesth 2015;29:146-8. 